\title{
Functional Connectivity from the Amygdala to the Hippocampus Grows Stronger after Stress
}

\author{
Supriya Ghosh, ${ }^{1}$ T. Rao Laxmi, ${ }^{2}$ and Sumantra Chattarji ${ }^{1}$ \\ ${ }^{1}$ National Centre for Biological Sciences, Bangalore 560065, India, and ${ }^{2}$ Department of Neurophysiology, National Institute of Mental Health and \\ Neurosciences, Bangalore 560029, India
}

The cellular and molecular effects of stress on the amygdala are strikingly different compared with those in the hippocampus. Previous findings on stress-induced plasticity were based primarily on postmortem analysis within individual areas. However, little is known about how stress affects dynamic changes and interactions in neuronal activity between the two areas. Hence, we simultaneously monitored in vivo activity of neuronal populations located in hippocampal areas CA1 and CA3 and the lateral amygdala (LA) in rats during and after chronic immobilization stress. The amplitude of auditory-evoked potentials (AEPs) in the hippocampus increased transiently only after a single $2 \mathrm{~h}$ stress but not when it was repeated for $10 \mathrm{~d}$. In contrast, both acute and chronic stress caused a persistent increase in AEPs in the LA. Chronic stress also elicited a sustained increase in the LA but a decrease in the hippocampus in the evoked power of gamma and beta frequencies. Moreover, beta and gamma synchrony was reduced between areas CA1 and CA3 but enhanced between the LA and hippocampus after chronic stress. Granger causality spectra revealed a strong directional influence from the LA to area CA1 that persisted throughout and even $10 \mathrm{~d}$ after chronic stress. However, directional coupling from hippocampal area CA3 to CA1 became weaker at the end of chronic stress. Thus, our findings suggest that the growing dominance of amygdalar activity over the hippocampus during and even after chronic stress may contribute to the enhanced emotional symptoms, alongside impaired cognitive function, seen in stressrelated psychiatric disorders.

\section{Introduction}

Prolonged stress leads to a range of behavioral abnormalities. Interestingly, some of the key emotional and cognitive symptoms of stress disorders are quite divergent. This is evident in the contrasting effects of chronic stress on the structure and function of two brain areas critically involved in learning and memory: the hippocampus and amygdala. Accumulating evidence from animal models shows that stress impairs hippocampal function at multiple levels of neural organization. Chronic stress causes dendritic atrophy (McEwen, 1999) and loss of spines (Pawlak et al., 2005) and suppresses long-term potentiation (LTP) (Pavlides et al., 2002) in the hippocampus. These detrimental stress effects at the molecular and cellular levels are believed to impair hippocampal learning and memory (Conrad, 2010), but chronic stress triggers opposite effects in the basolateral amygdala (BLA) by strengthening the structural basis of synaptic connectivity through dendritic growth and spinogenesis (Roozendaal et al., 2009). Repeated stress also enhances LTP in the BLA and facili-

\footnotetext{
Received Feb. 11, 2013; revised March 12, 2013; accepted March 14, 2013.

Author contributions: S.G., L.T.R., and S.C. designed research; S.G. performed research; S.G. analyzed data; S.G. and S.C. wrote the paper.

This work was supported by research funds from the National Centre for Biological Sciences and International Senior Research Fellowship GR 0701339 MA (S.C.) from the Wellcome Trust. We thank Dr. Rishikesh Narayanan for helpful comments and discussions.

The authors declare no competing financial interests.

Correspondence should be addressed to Prof. Sumantra Chattarji, National Center for Biological Sciences, Bangalore 560065, India. E-mail: shona@ncbs.res.in.

DOI:10.1523/JNEUROSCI.0638-13.2013

Copyright $\odot 2013$ the authors $\quad 0270-6474 / 13 / 337234-11 \$ 15.00 / 0$
}

tates fear and anxiety-like behavior (Roozendaal et al., 2009). Notably, morphological changes elicited by stress differ not only with spatial location but also over time. For instance, $10 \mathrm{~d}$ of immobilization stress elicits BLA dendritic hypertrophy lasting up to $21 \mathrm{~d}$ after stress (Vyas et al., 2004). In contrast, dendritic atrophy in hippocampal area CA3 reverses within 7-10 d after stress (Luine et al., 1994). Together, these animal studies have identified key spatial and temporal features of stress-induced plasticity in the amygdala that are distinct from the hippocampus.

Although the above findings present a powerful experimental framework for animal models of stress, they also underscore some unresolved issues. First, these findings were derived primarily from postmortem snapshots of morphological and molecular changes within individual brain areas after the end of stress. However, little is known about how stress modulates interactions between neuronal assemblies distributed across the hippocampus and amygdala over the course of stress. Second, fearconditioning increases synchronization of theta-frequency activity in the LA and CA1 (Seidenbecher et al., 2003). Stress enhances both cued and contextual fear memory despite having contrasting effects on the two structures (Shors et al., 1992; Conrad et al., 1999). This raises questions about how synchronized oscillatory activity is modulated between these two areas differentially affected by stress. Finally, in previous studies, stress-induced plasticity in different brain regions was often characterized as stand-alone effects intrinsic to individual structures. However, there are extensive anatomical interconnections between the hippocampus and amygdala (Pitkänen et al., 2000). 
A

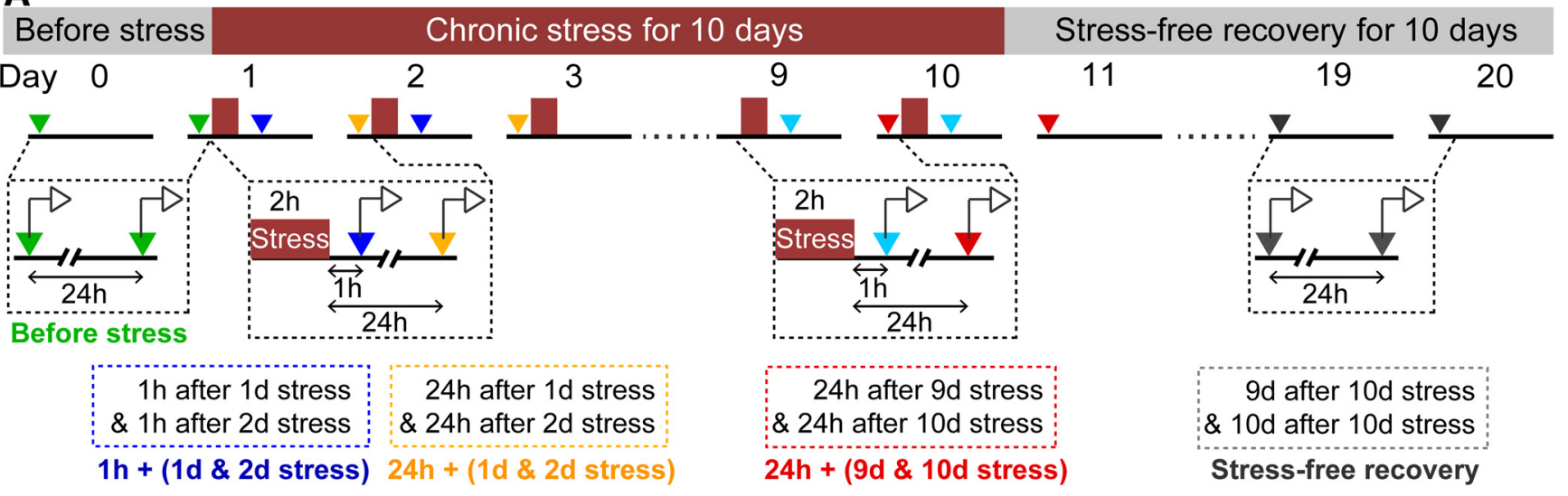

B

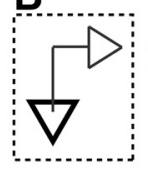

AEP recording
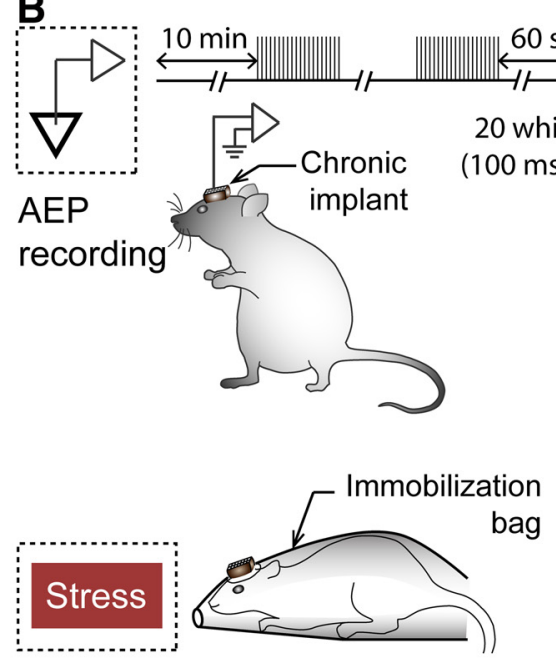

C

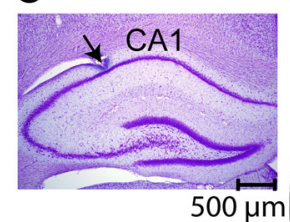

20 white-noise (100 ms) at $1 \mathrm{~Hz}$
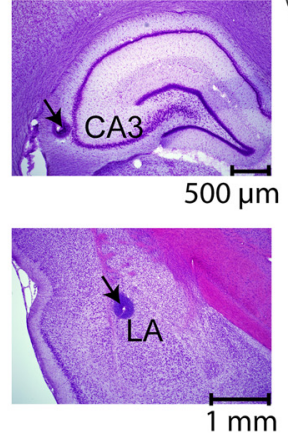

D $\quad-2.92 \mathrm{~mm}$

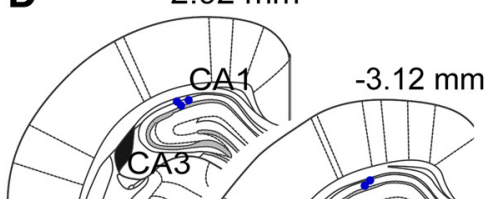
$-3.36 \mathrm{~mm}$

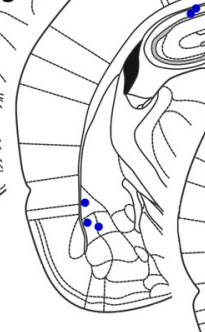

\& 10d after 10d stress

Stress-free recovery

Figure 1. Chronic in vivo recordings of $A E P$ s simultaneously from hippocampal areas $C A 1$ and $C A 3$ and the (LA) in the same animal before, during, and after chronic stress. $A$, Experimental design and time course for AEP recordings in hippocampal areas CA1 and CA3 and the LA from freely behaving rats before, during, and after $10 \mathrm{~d}$ CIS. B, Top, AEPs were recorded after 10 min of acclimatization in a standard home cage inside a sound-attenuating chamber. In every recording session, there were three trains of 20 white-noise pulses/train at $1 \mathrm{~Hz}$. Each of the white-noise pulses was for $100 \mathrm{~ms}(50 \mathrm{~dB})$. The intertrain intervals were $60 \mathrm{~s}$ long. Bottom, CIS consisted of $2 \mathrm{~h}$ complete immobilization in a plastic immobilization bag for 10 consecutive days. C, D, Histological verification of recording sites in hippocampal areas $C A 1$ and $C A 3$ and the $L A$. Representative photomicrographs of brain sections from an experimental rat stained with cresyl violet $(0.2 \%)$ showing the lesion spots corresponding to the electrode tips (pointed by arrows) in CA1 (top), CA3 (middle), and LA (bottom), respectively. Schematic of coronal rat brain sections (Paxinos and Watson, 2005) indicating placements of the electrode tips (blue dots, $\boldsymbol{D})$ within dorsal hippocampal areas CA1 and CA3 and the LA from all the experimental rats $(n=7)$.

This raises the intriguing possibility that some of the stressinduced changes triggered in one brain area may, at least in part, influence changes in other areas. Indeed, behavioral and pharmacological evidence suggests that the amygdala may mediate certain stress effects on hippocampal LTP and memory (Kim et al., 2001, 2005). These findings highlight the need to examine the potential impact of stress on directional influences between the hippocampus and amygdala. Therefore, we monitored the spatiotemporal dynamics of neuronal activity in hippocampal areas CA1 and CA3 and LA in freely moving rats using multielectrode in vivo recordings before, during, and after chronic immobilization stress (CIS).

\section{Materials and Methods}

Animals

Male Wistar rats (7-10 weeks old) were maintained in a 14/10 h light/ dark cycle with food and water ad libitum and housed in a group of two to three per cage in a climate-controlled colony room. All experimental protocols were conducted in accordance with Committee for the Purpose of Control and Supervision of Experiments on Animals guidelines of the Government of India and approved by the Institutional Animal
Ethics Committee, National Centre for Biological Sciences (Bangalore, India).

\section{Surgery}

Rats were anesthetized with a combination of xylazine-ketamine (10 and $80 \mathrm{mg} / \mathrm{kg}$, i.p., respectively). Teflon-coated stainless-steel microwires (50 $\mu \mathrm{m}$; A-M Systems) were chronically implanted in the LA and hippocampal areas CA1 and CA3 under stereotaxic (David Kopf Instruments) guidance [bregma in $\mathrm{mm}$ : LA: anteroposterior (AP) +3.3, mediolateral (ML) 5.5, and dorsoventral (DV) 8.2; CA1: AP +3.3, ML 2.5, and DV 2.4; CA3: AP +3.6, ML 3.8, and DV 3.8 (Paxinos and Watson, 2005)].

\section{Stress protocol}

Each stress episode consisted of complete immobilization $(2 \mathrm{~h} / \mathrm{d}$ for 10 consecutive days, 10:00 A.M. to 12:00 P.M.) in rodent immobilization bags (Vyas et al., 2002), with chronically implanted recordings electrodes intact, without any access to food or water (Fig. 1B).

In vivo electrophysiological recordings

After postsurgical recovery for $10 \mathrm{~d}$, rats were placed in a rat's home cage kept inside a sound-attenuated chamber (Coulbourn Instruments) for electrophysiological recordings. Auditory-evoked potentials (AEPs) 
were recorded by connecting the microelectrodes to a unit gain buffer head stage (HS-36-Flex; Neuralynx) and a data acquisition system Digilynx (Neuralynx). Neural data were amplified $(500 \times)$ and acquired at a sampling rate of $4 \mathrm{kHz}$, followed by a bandpass filter (1-2000 Hz) using Cheetah data acquisition software (Neuralynx).

\section{Experimental design}

A single session of recording was performed every day (9:00 A.M. to 10:00 A.M.) simultaneously from areas CA1, CA3, and LA in the same animal for 21 consecutive days (Fig. $1 \mathrm{~A}, B$ ), starting with the day before the beginning of chronic stress (day 0 ), followed by 10 consecutive days of CIS (days 1-10; Fig. 1A) $1 \mathrm{~h}$ immediately before daily stress, and ending with another $10 \mathrm{~d}$ of stress-free recovery (days 11-20; Fig. 1A). In addition, recordings were also obtained $1 \mathrm{~h}$ after the end of each $2 \mathrm{~h}$ stress (Fig. 1A). AEP recordings started $10 \mathrm{~min}$ after the animal was acclimatized to the recording chamber. In each session, rats were presented with three trains of 20 white-noise pulses/train $(100 \mathrm{~ms}, 1 \mathrm{~Hz}, 60 \mathrm{~s}$ intertrain interval, $50 \mathrm{~dB}$; Fig. 1B). To analyze the temporal progression of the relative impact of stress- during the early versus later stages of chronic stress-AEPs recorded at multiple time points were averaged into five separate blocks. A single value for each of these blocks was calculated by averaging AEPs for the same time point after stress on 2 consecutive days (total of 120 trials) at the beginning, end, or after chronic stress. These five blocks, with their respective color coding shown in Figure $1 \mathrm{~A}$, are as follows: (1) Before stress, average of AEPs from two initial recordings on day 0 and day 1 (green); (2) $1 \mathrm{~h}+(1 \mathrm{~d} \& 2 \mathrm{~d}$ stress), average of AEPs recorded $1 \mathrm{~h}$ after day 1 and day 2 of chronic stress (blue); (3) $24 \mathrm{~h}+(1$ d \& 2 d stress), average of AEPs recorded $24 \mathrm{~h}$ after day 1 and day 2 of chronic stress (orange); (4) $24 \mathrm{~h}+(9 \mathrm{~d} \& 10 \mathrm{~d}$ stress), average of AEPs recorded $24 \mathrm{~h}$ after day 9 and day 10 of stress (Fig. $1 A$, red); (5) Stress-free recovery, average of AEPs recorded 9 and $10 \mathrm{~d}$ after the end of chronic stress (gray). This scheme of comparisons across time was used for all other parameters (Figs. 2-7).

\section{Data analyses}

$A E P s$. Averaged AEPs were quantified by measuring the amplitude, slope, and latency to peak of the negative potentials (Rogan et al., 1997; Tang et al., 2003; Brockhaus-Dumke et al., 2008). The amplitude and slope were measured by the difference between the maxima (see Fig. $2 A-C$, dot) after the onset of the response and the negative peak (see Fig. $2 A-C$, arrow) (hippocampus, $40-60 \mathrm{~ms}$; LA, 20-30 ms). The latency was measured as the time at which negative peak occurs.

Time-frequency analyses. Analysis of event-related changes in spectral power and phase consistency were performed using continuous wavelet transformation (MATLAB) on the AEPs. Complex Morlet wavelets were used to compute phase and the amplitude of evoked responses within a frequency range from 2 to $120 \mathrm{~Hz}$ in steps of $0.1 \mathrm{~Hz}$ (Makeig et al., 2004; Li et al., 2007). The bandwidth parameter and center frequency of the mother wavelet were 2 and $1 \mathrm{~Hz}$, respectively. The continuous wavelet transformation of a signal $x(t)$ is defined as $W_{x}(\tau, s)=\left\langle x, \psi_{\tau, s}\right\rangle$ $=\int_{-\infty}^{+\infty} x(t) \frac{1}{\sqrt{s}} \psi^{*}\left(\frac{t-\tau}{s}\right) d t$, where $\psi^{*}(t)$ is the complex conjugate of the wavelet function $\psi(t), s$ is the dilation (scale) parameter; and $\tau$ is the translation (location) parameter of the wavelet. The complex Morlet wavelet function is defined as $\psi(t)=\frac{1}{\sqrt{\pi f_{b}}} e^{\frac{-t^{2}}{f_{b}}} e^{\text {i2 } 2 \pi f_{c} t}$, where $f_{b}$ is the bandwidth parameter, $f_{c}$ is the center frequency of the wavelet, and $\psi\left(\frac{t-\tau}{S}\right)$ is the time-shifted and scaled version of the mother wavelet $\psi(t)$. The wavelet power of the time series $x(t)$ in the timescale space is expressed (in decibels) as $P_{x}(\tau, s)=10 \log \left(\left|W_{x}(\tau, s)\right|^{2}\right)$. The frequencies associated with the scales are expressed as, $f=\frac{f_{c} \times F_{s}}{S}$, where $F_{s}$ is the sampling rate, and the difference in power was computed by subtracting baseline values $(-100$ to $0 \mathrm{~ms}$ ) for every frequency. For the population comparison, auditory evoked powers between 25 and $100 \mathrm{~ms}$ from the tone onset were averaged. Dominant frequency was computed by determining the frequency of maximum power. Two-way repeated-measures ANOVA [two withinsubject factors, recording blocks (five levels) and frequencies $(10-40 \mathrm{~Hz}$ with step size of $0.1 \mathrm{~Hz} ; 301$ levels)] was used to compare the frequency ranges over which the evoked powers were most affected by stress in all the three brain areas. Overall powers around the dominant frequencies (16-36 Hz in CA1 and CA3; $28-38 \mathrm{~Hz}$ in LA) were compared across recording sessions.

Phase synchrony. To detect the phase synchrony between two distant brain areas, single-trial phase locking values (sPLV), a measure of the stability of the instantaneous phase differences, were computed (Lachaux et al., 2000). sPLV between two signals $x(t)$ and $y(t)$ was obtained by averaging of the instantaneous phase differences over a moving time

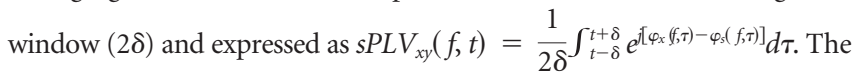
phase difference was computed from the wavelet coefficients of the signals, $e^{\left.i \varphi_{x}(f, t)-\varphi_{x}(f, t)\right]}=\frac{W_{x}(f, t) W_{y}^{*}(f, t)}{\left|W_{x}(f, t) W_{y}(f, t)\right|^{\circ}}$. Hippocampus-amygdala sPLV during the AEP trials was computed by using a $10 \mathrm{~ms}$ moving window and then averaged over trials. For population comparison, sPLV ( $z$-score converted) was averaged across beta/gamma frequency range (CA1CA3, 30-34 Hz; CA1-LA, 26-36 Hz) and compared over a period of $20-80 \mathrm{~ms}$ from tone onset, across rats using two-way repeated-measures ANOVA (factor 1, recording blocks; factor 2, time from tone onset).

Granger causality. Granger spectral causality within CA1-CA3-LA network was performed using BSMART (Cui et al., 2008) MATLAB toolbox. According to Wiener and Beckenbach (1956) and Granger (1969), if the variance of the autoregressive prediction error in a linear regression model of a time series $x_{1}(t)$ at the present time is reduced by inclusion of past measurements from a second time series $x_{2}(t)$, then $x_{1}(t)$ is said to have a causal influence from $x_{2}(t)$. To estimate the relative strengths of directional influences between two structures, time series were modeled as bivariate autoregressive models. For a pair of structures, the bivariate autoregressive model for $X(t)=\left\{x_{1}(t), x_{2}(t)\right\}$ can be expressed as $\Sigma_{j=0}^{m} A_{j} X(t-j)=E(t)$, where $m$ is the model order, $A_{j}$ is the $2 \times 2$ coefficient matrices, and $E(t)$ is the residual error with a covariance matrix $\Sigma$. The model coefficients $A_{j}$ and $\Sigma$ were estimated from LevinsonWiggins-Robinson algorithm (Ding et al., 2000). The spectral matrix was computed from the model coefficients $A_{j}$ and $\Sigma, S(f)=H(f) \Sigma H^{\star}(f)$, where $H(F)$ is the transfer function expressed as $H(f)=\left(\sum_{j=0}^{m} A_{j} c^{-2 \pi i f}\right)^{-1}$, and ${ }^{*}$ corresponds to transposition and complex conjugation. According to Geweke's (Geweke, 1982) formulation, Granger causality in the spectral domain for each direction of influence for the two time series ( 1 and 2) can be defined as

$$
\begin{aligned}
& I_{2 \rightarrow 1}=-\ln \left|1-\frac{\left(\Sigma_{22}-\frac{\Sigma_{12}^{2}}{\Sigma_{11}}\right)\left|H_{12}(f)\right|^{2}}{S_{11}(f)}\right| ; \\
& I_{1 \rightarrow 2}=-\ln \left|1-\frac{\left(\Sigma_{11}-\frac{\Sigma_{12}^{2}}{\Sigma_{22}}\right)\left|H_{21}(f)\right|^{2}}{S_{22}(f)}\right|,
\end{aligned}
$$

where the power spectrum of time series $x_{1}(t)$ and $x_{2}(t)$ are, respectively, $S_{11}(f)$ and $S_{22}(f)$, and $\Sigma_{11}, \Sigma_{22}, \Sigma_{12}$ are the elements of the matrix $\Sigma$. Artifact free trials of the AEPs of $500 \mathrm{~ms}$ long (from -200 to $300 \mathrm{~ms}$ with respect to tone onset), from all the three structures areas CA1, CA3, and LA, were subsampled to $509 \mathrm{~Hz}$ and preprocessed. The ensemble mean was subtracted from each single-trial AEP time series and divided by the $\mathrm{SD}$ to remove first-order nonstationarity and to get an ensemble of single-trial time series considered as zero mean stochastic process. To analyze the temporal evolution of the directional influence, causality was estimated in successive windows of $50 \mathrm{~ms}$ advanced by $2 \mathrm{~ms}$. The model order for the bivariate autoregressive model was chosen as 15 based on the minimal values of the Akaike information criterion (Ding et al., 2000; Cui et al., 2008). The suitability of the model for our dataset was confirmed by (1) low whiteness $(<1.5 \%)$, a measure of white model residuals and absence of temporal correlations, (2) negative stability index, a measure of the stability of the fitted model, and (3) high consistency $(>90 \%)$, a measure of the correlation structure indicating the fraction of variance captured by the model (Ding et al., 2000; Cui et al., 2008). The 
Granger spectral causality coefficients between a pair of brain regions for each animal at a particular time point were compared with the significance limit obtained from a surrogate datasets $(n=100)$. One such dataset (60 trials) was composed of trials with time shifts between 100 and $400 \mathrm{~ms}$ (in random) for every trail and then directional coefficients were computed. These coefficients $(n=100)$ were used to get significance limit (95\% confidence interval, normal fit). The latency of a given directional influence was estimated as the time at which the first of the five consecutive time points $(10 \mathrm{~ms})$ was significantly higher than the reverse direction for a pair of structures (paired $t$ test, $p<0.05$ ).

Volume conduction. The extent of volume conduction in the local field potentials (LFPs) recorded from three brain structures, areas CA1, CA3 and LA, were estimated using three different measures: (1) crosscorrelations of the amplitude signals, (2) cross-correlations of the phase signals, and (3) phase lag index (PLI) (Daffertshofer and Stam, 2007). The PLI is a measure of the symmetry of phase distributions, which is the probability (bound between 0 and 1 ) of occurrence of the deviation of the relative phase from 0 and $\pm \pi$ and given as PLI $=\left|\left\langle e^{i\left(\varphi_{x}-\varphi_{y}\right)}\right\rangle\right|$, where, $\varphi_{x}$ and $\varphi_{y}$ are the phases of the two time series $x$ and $y$. The large value of the PLI implies the presence of correlations without contribution from volume conduction. Spontaneously recorded LFPs of $200 \mathrm{~s}$ duration (100 s per session) collected from every rat before stress and then instantaneous amplitude and phase signals were obtained by Hilbert transformation. The cross-correlations of the amplitudes and phases were computed from $10 \mathrm{~s}$ epochs from these $200 \mathrm{~s}$ (total 20) spontaneous LFPs, for all pairs of brain structures (CA1, CA3 and LA) and for every rat. PLI was estimated from full 200-s-long LFPs for every rats and compared with zero using two-tailed $t$ test.

Statistical analyses. Population comparison of stress-induced effects on AEP amplitudes, slopes, latencies, dominant frequencies, and beta/ gamma band power were analyzed using one-way repeated-measures ANOVA, followed by Fisher's least significant difference post hoc comparison. sPLVs were compared using two-way repeated-measures ANOVA. Directional flow was compared using paired Student's $t$ test of the Granger spectral causality coefficients within successive $25 \mathrm{~ms}$ time windows between two directions for a pair of brain areas.

\section{Histology}

Placements of the recording electrodes were verified after completion of the experiments using standard histological methods. Rats were deeply anesthetized (ketamine-xylazine, 100 and $20 \mathrm{mg} / \mathrm{kg}$, i.p., respectively) and electrolytic lesions were made to mark the recording sites by passing anodal currents $(20 \mu \mathrm{A}$ for $20 \mathrm{~s}$ ). The animals were then perfused transcardially with ice-cold saline, followed by $10 \%$ formalin solution and $10 \%$ sucrose in saline sequentially. Cryoprotected brains were sectioned (coronal, $60 \mu \mathrm{m}$ ) using the cryostat (Leica Microsystems) and mounted on gelatin-coated glass slides. Sections were stained with $0.2 \%$ cresyl violet solution $\left(60^{\circ} \mathrm{C}\right)$ to verify the placement of the electrode tip (Fig. $1 C, D)$.

\section{Results}

In our experimental paradigm, we focused on the effects of acute and chronic stress on the activity of neuronal populations located in three specific subregions of the amygdala-hippocampal network that are known to exhibit contrasting patterns of stressinduced plasticity in rodents: CA1 and CA3 in the hippocampus and the LA (Fig. 1; see Materials and Methods). To this end, rats were subjected to $2 \mathrm{~h}$ of immobilization stress per day for 10 consecutive days, and AEPs were monitored simultaneously in the areas CA1, CA3 and LA in the same animal at different time points before, during, and after the $10 \mathrm{~d}$ stress paradigm (Fig. 1). Consistent with previous reports, trains of white-noise pulses (see Materials and Methods) reliably elicited negative-deflecting potentials in all three areas (Fig. 2A-C) (Rogan et al., 1997; Tang et al., 2003).
Acute exposure to stress enhances AEP amplitudes in both the hippocampus and LA, but they differ in terms of post-stress recovery

We first examined the impact of a single episode of $2 \mathrm{~h}$ immobilization stress on AEPs recorded $1 \mathrm{~h}$ and $1 \mathrm{~d}$ later (Fig. 2). AEP amplitudes in all three areas-CA1, CA3 and LA-were significantly affected by stress (CA1, $F_{(9,54)}=5.17, p<0.001$; CA3, $F_{(9,54)}=6.45, p<0.001 ; \mathrm{LA}, F_{(9,54)}=2.77, p<0.01$; Fig. $\left.2 A-C\right)$. This single exposure to acute stress caused a significant increase in AEP amplitudes $1 \mathrm{~h}$ later relative to pre-stress baseline values in both hippocampal areas CA1 (264 $\pm 48 \%, p<0.001$; Fig. $2 A$, blue) and CA3 (214 $\pm 37 \%, p<0.01$; Fig. $2 B$, blue). Acute stress also enhanced AEP amplitude in the LA at the same $1 \mathrm{~h}$ time point (195 $\pm 24 \%, p<0.01$; Fig. $2 C$, blue). Notably, the increase in AEP amplitude persisted in the LA even $1 \mathrm{~d}$ after the acute stress (177 $\pm 28 \%, p<0.05$; Fig. $2 C$, orange). In contrast, $1 \mathrm{~d}$ later, AEPs in areas CA1 and CA3 underwent a significant decrease relative to the peak values seen $1 \mathrm{~h}$ after stress $(p<0.05$; Fig. $2 A, B$, orange). Thus, although the increase in AEPs after the first episode of acute stress (i.e., after the first day of chronic stress) was similar across the hippocampus and amygdala $1 \mathrm{~h}$ later, they differed in terms of their persistence when measured $24 \mathrm{~h}$ later. The same temporal profile of modulation in AEP amplitudes was also seen after a second episode of acute stress (i.e., after the second day of chronic stress), a robust short-term increase $1 \mathrm{~h}$ later in all three areas (CA1, $296 \pm 36 \%, p<0.001$; CA3, $275 \pm$ $20 \%, p<0.001$; LA, $190 \pm 16 \%, p<0.01$; Fig. $2 A-C$, blue) that persisted to a greater extent in the LA compared with areas CA1 and CA 3 at the $24 \mathrm{~h}$ time point (CA1, $179 \pm 34 \%, p>0.05$; CA3, $147 \pm 21 \%, p>0.05$; LA, $163 \pm 15 \%, p<0.05$; Fig. $2 A-C$, orange).

\section{Repeated exposure to the same stress has no effect on AEP amplitudes in the hippocampus but leads to a persistent increase in the LA}

Additional differences in the impact of the $2 \mathrm{~h}$ session of stress on AEPs, both in terms of brain regions and temporal features, emerged when the same stressor was repeated for 10 consecutive days (i.e., CIS). Strikingly, unlike the first and second episodes of acute stress, exposure to the same $2 \mathrm{~h}$ stress for the last $2 \mathrm{~d}$ of the $10 \mathrm{~d}$ chronic stress protocol (i.e., for the 9 th and 10 th times) failed to cause any increase in AEP amplitudes at the $1 \mathrm{~h}$ post-stress time points in either area CA1 or CA3 ( $p>0.05$; Fig. $2 A, B)$. Furthermore, $24 \mathrm{~h}$ after the 9th and 10th episodes of stress, the AEP amplitudes were not different in these areas from pre-stress baseline values $(p>0.05$; Fig. $2 A, B)$. In contrast, in the LA, the amplitude of AEP continued to be significantly greater at both 1 and $24 \mathrm{~h}$ after the 9 th and 10th days of chronic stress $(1 \mathrm{~h}+9 \mathrm{~d}$ stress, $190 \pm 32 \%, p<0.01 ; 1 \mathrm{~h}+10 \mathrm{~d}$ stress, $181 \pm 36 \%$, $p<$ $0.05 ; 24 \mathrm{~h}+9 \mathrm{~d}$ stress, $165 \pm 19 \%, p<0.05 ; 24 \mathrm{~h}+10 \mathrm{~d}$ stress, $170 \pm 25 \%, p<0.05$; Fig. $2 C$ ). Thus, in the hippocampus, the same $2 \mathrm{~h}$ episode of stress facilitated AEPs immediately $(1 \mathrm{~h})$ after the stress during the first two but not the last two days of the $10 \mathrm{~d}$ chronic stress paradigm. Conversely, the enhancing effect of stress on AEPs in the LA remained undiminished until the end of the chronic stress protocol. Finally, despite these differences, AEP amplitudes returned to pre-stress baseline levels both in areas CA1 and CA3 and the LA after an additional $10 \mathrm{~d}$ of stress-free recovery (Fig. $2 A-C$, gray). Figure $2 D-F$ summarizes the abovementioned effects on AEP amplitudes in both hippocampal areas CA1 $\left[F_{(4,24)}=14.17, p<0.001 ; 1 \mathrm{~h}+(1 \mathrm{~d} \& 2 \mathrm{~d}\right.$ stress $), p<0.001$; $24 \mathrm{~h}+(1 \mathrm{~d} \& 2 \mathrm{~d}$ stress $), p<0.05$; Fig. $2 D]$ and CA3 $\left[F_{(4,24)}=\right.$ 13.72, $p<0.001 ; 1 \mathrm{~h}+(1 \mathrm{~d} \& 2 \mathrm{~d}$ stress $), p<0.001$; Fig. $2 E]$ and 


\section{A}

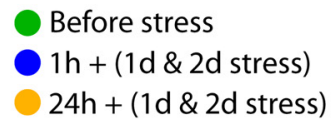

$1 \mathrm{~h}+(9 \mathrm{~d} \& 10 \mathrm{~d}$ stress)

$24 \mathrm{~h}+(9 \mathrm{~d} \& 10 \mathrm{~d}$ stress)

Stress-free recovery
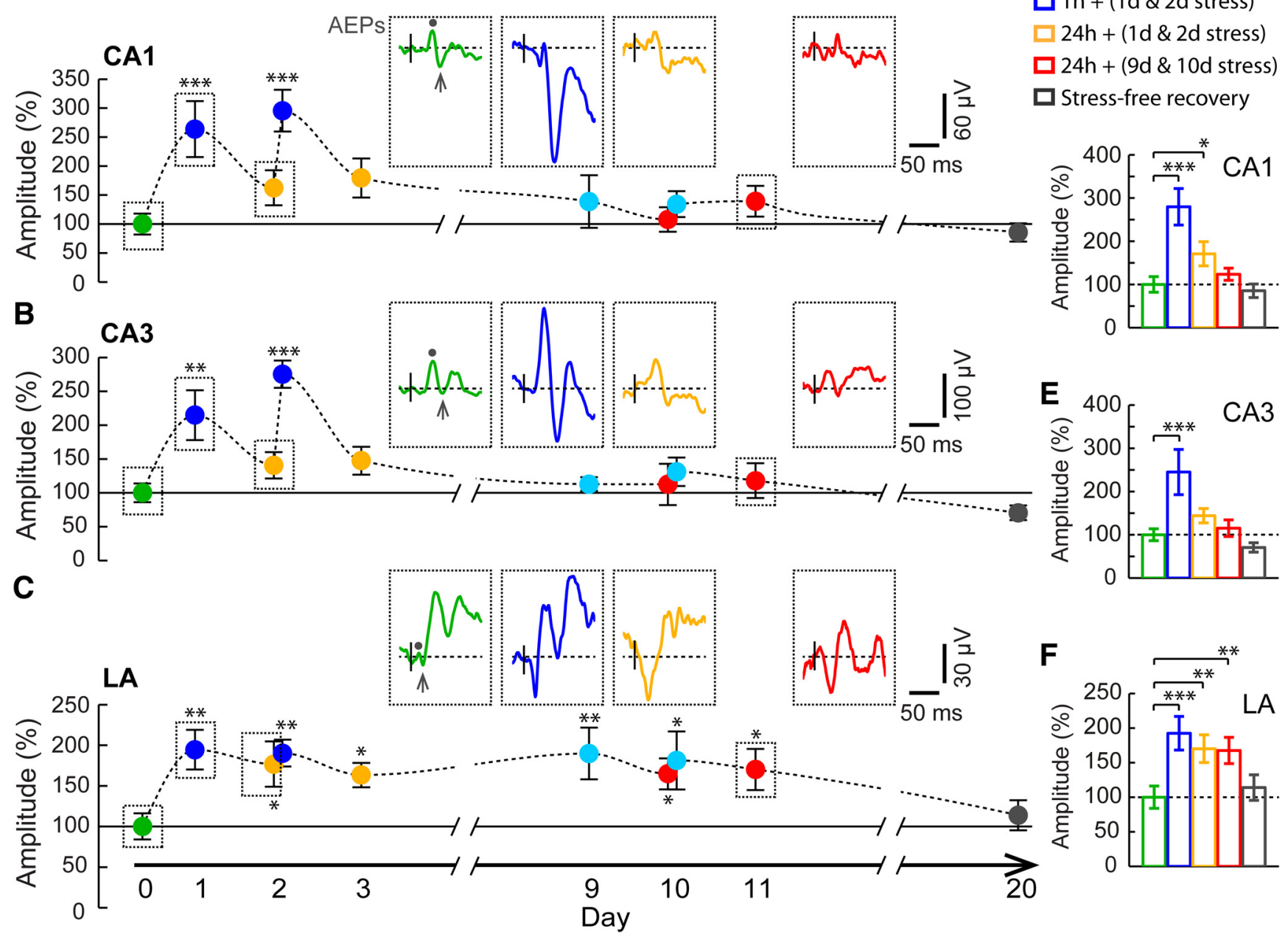

Figure 2. Contrasting effects of chronic stress on AEPs in the hippocampus and amygdala. $\boldsymbol{A}-\boldsymbol{C}$, Time course of changes in mean amplitude of AEPs ( $n=7$ ), normalized to pre-stress baseline values, recorded in CA1 $(\boldsymbol{A}), C A 3(\boldsymbol{B})$, and LA (C). Insets, Representative AEP traces recorded in all three areas before stress (green), $1 \mathrm{~h}$ (blue) and $24 \mathrm{~h}$ (orange) after the first day of chronic stress, and $24 \mathrm{~h}$ (red) after the 10th day of chronic stress. AEP amplitudes were quantified by measuring the difference of the peak negative deflection (arrow) relative to first positive peak (dot). Stress significantly enhanced AEP amplitudes in CA1, CA3, and LA $1 \mathrm{~h}$ after the first and second days of stress but differed in their temporal persistence with repeated exposure. $D-F$, Mean AEP amplitudes $(n=7)$ compared across five blocks of time, as depicted in Figure $1 A$ (see Materials and Methods). In areas CA1 (D) and CA3 (E), AEPs were enhanced only during the early stages of chronic stress. In contrast, LA $(\boldsymbol{F})$ exhibited a persistent increase for all $10 \mathrm{~d}$. Error bars indicate \pm SEM. ${ }^{*} p<0.05,{ }^{* *} p<0.01,{ }^{* * *} p<0.001$.

Table 1. Slopes (mean \pm SEM) and latencies (mean \pm SEM) of AEPs recorded in hippocampal areas CA1 and CA3 and the LA before, during, and after chronic stress

\begin{tabular}{|c|c|c|c|c|c|c|}
\hline & \multicolumn{3}{|l|}{ Slope (\%) } & \multicolumn{3}{|l|}{ Latency (ms) } \\
\hline & CA1 & CA3 & $\mathrm{LA}$ & CA1 & CA3 & $\mathrm{LA}$ \\
\hline Before stress & $100 \pm 18.0$ & $100 \pm 14.9$ & $100 \pm 16.0$ & $46.8 \pm 0.9$ & $47.9 \pm 1.5$ & $20.5 \pm 1.2$ \\
\hline $1 \mathrm{~h}+(1 \mathrm{~d} \& 2 \mathrm{~d}$ stress $)$ & $277.4 \pm 43.5^{* * *}$ & $247.9 \pm 53.2^{* * *}$ & $188.6 \pm 19.5^{* * *}$ & $47.3 \pm 1.0$ & $50.3 \pm 1.5$ & $17.6 \pm 1.0$ \\
\hline $24 \mathrm{~h}+(1 \mathrm{~d} \& 2 \mathrm{~d}$ stress $)$ & $172.4 \pm 27.4^{*}$ & $149.9 \pm 17.3$ & $170.0 \pm 19.8^{* *}$ & $46.9 \pm 1.0$ & $48.7 \pm 2.0$ & $20.6 \pm 1.2$ \\
\hline $24 \mathrm{~h}+(9 \mathrm{~d} \& 10 \mathrm{~d}$ stress $)$ & $123.8 \pm 13.9$ & $118.6 \pm 19.6$ & $165.9 \pm 19.0^{* *}$ & $45.4 \pm 0.3$ & $49.2 \pm 1.8$ & $21.2 \pm 1.5$ \\
\hline Stress-free recovery & $85.1 \pm 15.6$ & $72.7 \pm 11.0$ & $110.1 \pm 19.6$ & $45.6 \pm 1.0$ & $45.9 \pm 1.5$ & $21.9 \pm 2.7$ \\
\hline
\end{tabular}

${ }^{*} p<0.05,{ }^{* *} p<0.01$, and ${ }^{* * *} p<0.001$, compared with the values before stress.

the LA $\left[F_{(4,24)}=8.56, p<0.001 ; 1 \mathrm{~h}+(1 \mathrm{~d} \& 2 \mathrm{~d}\right.$ stress $), p<$ $0.001 ; 24 \mathrm{~h}+(1 \mathrm{~d} \& 2 \mathrm{~d}$ stress $), p<0.01 ; 24 \mathrm{~h}+(9 \mathrm{~d} \& 10 \mathrm{~d}$ stress), $p<0.01$; Fig. $2 F$ ] for all five blocks of time points before, during, and after chronic stress. Similar to the AEP amplitudes, AEP slopes were also affected by chronic stress in the same way in all three brain areas (Table 1). At the beginning of chronic stress, AEP slopes in CA1 and CA3 transiently increased [CA1, $F_{(4,24)}=$ 13.72, $p<0.001 ; 1 \mathrm{~h}+(1 \mathrm{~d} \& 2 \mathrm{~d}$ stress $), p<0.001 ; 24 \mathrm{~h}+(1 \mathrm{~d}$
$\& 2 \mathrm{~d}$ stress), $p<0.05$; CA3, $F_{(4,24)}=12.73, p<0.001 ; 1 \mathrm{~h}+(1$ $\mathrm{d} \& 2 \mathrm{~d}$ stress), $p<0.001$ ]. In contrast, stress facilitated the AEP slopes in LA in a persistent manner throughout the period of $10 \mathrm{~d}$ of chronic stress $\left[F_{(4,24)}=7.63, p<0.001 ; 1 \mathrm{~h}+(1 \mathrm{~d} \& 2 \mathrm{~d}\right.$ stress $)$, $p<0.001 ; 24 \mathrm{~h}+(1 \mathrm{~d} \& 2 \mathrm{~d}$ stress $), p<0.01 ; 24 \mathrm{~h}+(9 \mathrm{~d} \& 10 \mathrm{~d}$ stress), $p<0.01]$. The latencies of the evoked responses were not affected significantly by stress $\left(\mathrm{CA} 1, F_{(4,24)}=1.12, p=0.37\right.$; CA3, $F_{(4,24)}=2.04, p=0.12 ;$ LA, $F_{(4,24)}=1.94, p=0.13$; Table 1]. 

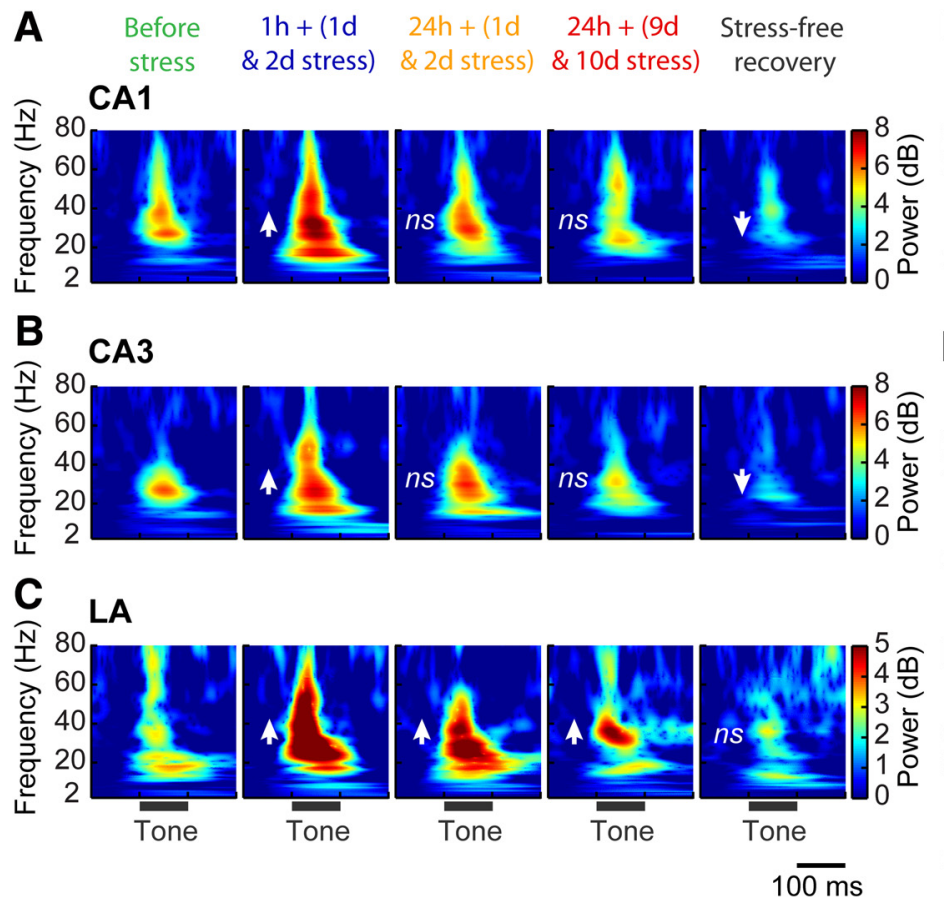

Figure 3. Persistent enhancement in the power of evoked beta/gamma oscillations in the LA but not the hippocampus during chronic stress. $\boldsymbol{A}-\boldsymbol{C}$, Average time-frequency evoked power spectrum $(n=7)$ in CA1 (A), CA3 (B), and LA ( $\boldsymbol{C}$ ) before, during, and after chronic stress (divided into 5 time blocks shown as columns). Absolute power was normalized to pre-tone (100 ms) values. Arrows indicate the direction of changes in the beta/gamma power. $\boldsymbol{D}-\boldsymbol{F}$, The average beta/gamma evoked power within 25-100 $\mathrm{ms}$ in $\mathrm{CA1}(\boldsymbol{D}), \mathrm{CA} 3(\boldsymbol{E})$, and LA $(\boldsymbol{F})$. Chronic stress caused a biphasic change in CA1 power $(16-36 \mathrm{~Hz}$; $\boldsymbol{D})$, with an immediate increase after stress [ $1 \mathrm{~h}+(1 \mathrm{~d} \& 2 \mathrm{~d}$ stress), $p<0.01]$ but a subsequent decrease during stress-free recovery $(p<0.01)$. Similarly biphasic modulation was seen in CA3 $(16-36 \mathrm{~Hz} ; \boldsymbol{E})$, a transient increase [1 $\mathrm{h}+(1 \mathrm{~d} \& 2 \mathrm{~d}$ stress); $p<0.05]$, followed by a delayed decrease (stress-free recovery, $p<0.01)$. However, power in LA $(28-38 \mathrm{~Hz})$ was persistently high during chronic stress [1 $\mathrm{h}+(1$ $\mathrm{d} \& 2 \mathrm{~d}$ stress), $p<0.01 ; 24 \mathrm{~h}+(1 \mathrm{~d} \& 2 \mathrm{~d}$ stress), $p<0.05 ; 24 \mathrm{~h}+(9 \mathrm{~d} \& 10 \mathrm{~d}$ stress), $p<0.05 ;(\boldsymbol{F})]$. ns, Not significant. Error bars indicate \pm SEM. ${ }^{*} p<0.05,{ }^{* *} p<0.01$.

Chronic stress leads to a delayed impairment of the power in beta/gamma oscillations in the hippocampus but a persistent enhancement in the LA

Sensory stimulus evoked oscillations in a range of frequency bands have been suggested to underlie changes in evoked potentials (Haenschel et al., 2000; Jung et al., 2006; Spencer et al., 2008). Thus, the mean power spectrum was computed for the averaged AEPs in all three brain areas before, during, and after chronic stress (Fig. 3). Before the beginning of stress, this analysis showed that auditory stimulation evoked oscillatory activity predominantly in the beta to gamma frequency bands in all three areas (Fig. 3A-C, first column). The impact of stress was further analyzed by calculating the average power for the three recording sites during a period of $25-100 \mathrm{~ms}$ from tone onset. Chronic stress had no effects on the dominant frequencies present in the AEPs in hippocampal areas CA 1 and CA3 $\left(\mathrm{CA} 1, F_{(4,24)}=0.9, p=\right.$ 0.47 ; CA3, $\left.F_{(4,24)}=2.03, p=0.12\right)$, whereas it was increased in the LA $\left(F_{(4,24)}=16.72, p<0.001\right)$ (Table 2). Moreover, evoked power was affected around the dominant frequencies in both areas CA1 and CA3 over a broader range of frequencies compared with the LA, in which there was a persistent increase during chronic stress within a narrower frequency range (Fig. $3 A-C$, Table 2). Hence, to ensure that our analysis was sufficiently inclusive despite these inherent differences between brain areas, we chose two different frequency bands-20 Hz (16-36 $\mathrm{Hz}$ ) for areas CA1 and CA3 and $10 \mathrm{~Hz}(28-38 \mathrm{~Hz})$ for LA-to compare the overall average values of the evoked powers over blocks of time points (Fig. 3D-F). There was a significant effect of

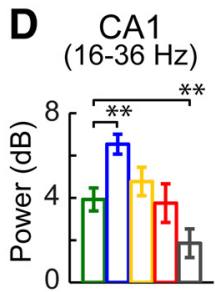

E $\mathrm{CA} 3$ $(16-36 \mathrm{~Hz})$
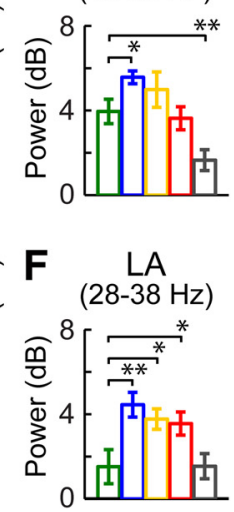

stress on the evoked powers in all three brain areas $\left(\mathrm{CA} 1, F_{(4,24)}=8.88, p<0.001\right.$; CA3, $F_{(4,24)}=8.69, p<0.001 ; \mathrm{LA}, F_{(4,24)}$ $=8.09, p<0.001)$ with an increase $1 \mathrm{~h}$ after the end of stress, averaged over the first and second day of stress [ $1 \mathrm{~h}+(1 \mathrm{~d} \&$ 2 d stress), Fig. $3 A-C$, second column; CA1, $p<0.01$; CA3, $p<0.05$; LA, $p<$ 0.01 , Fig. $3 D-F]$. This increase in power persisted in the LA but not in hippocampus, even after $24 \mathrm{~h}[24 \mathrm{~h}+(1 \mathrm{~d} \& 2 \mathrm{~d}$ stress), Fig. 3A-C, third column; LA, $p<$ 0.05 , Fig. $3 F$ ]. Furthermore, this enhancing effect in the LA persisted even after the end of the $10 \mathrm{~d}$ stress protocol $[24 \mathrm{~h}+(9 \mathrm{~d}$ $\& 10 \mathrm{~d}$ stress), Fig. $3 A$, fourth column; $p<$ 0.05 , Fig. $3 F]$. In contrast, the evoked power in areas CA1 and CA3 were not affected $24 \mathrm{~h}$ after the end of $10 \mathrm{~d}$ chronic stress (Fig. $3 A, B$ ). An additional difference between the amygdala and hippocampus became evident when the animals were allowed to recover for $10 \mathrm{~d}$ after the end of chronic stress. At this $10 \mathrm{~d}$ post-stress time point, the power in the CA 1 and CA 3 areas decreased relative to pre-stress values (stress-free recovery, Fig. $3 A, B$, fifth column; CA $1, p<0.01$; CA3, $p<0.01$, Fig. $3 D, E)$. However, power in the LA returned to pre-stress baseline levels (stress-free recovery, Fig. $3 C$, fifth column; $p>0.05$, Fig. $3 F)$. Finally, the evoked power of the beta/gamma oscillations were positively correlated with AEP amplitudes in all three areas (CA1, $r=0.77, p<0.001$; CA3, $r=0.56, p<0.001 ; \mathrm{LA}, r=0.45, p<$ $0.01)$.

Phase synchrony is reduced within the hippocampus but enhanced between the amygdala and hippocampus after chronic stress

Neuronal synchronization in distinct frequency bands have been recorded in many brain regions during sensory and memory processing. Moreover, the absence or disruption of such synchrony between brain areas has been reported in rodent models of cognitive dysfunction (Dzirasa et al., 2009; Uhlhaas and Singer, 2010). Thus, we next examined whether the enhanced beta/ gamma power was accompanied by an increase in phase synchrony between areas CA1 and CA3 and the LA. To this end, we quantified phase synchrony by means of sPLVs (Lachaux et al., 2000), a measure of variability of the phase differences over small time windows $(10 \mathrm{~ms})$ for simultaneously recorded pairs of AEPs from all three areas (see Materials and Methods). Oscillations in these frequencies during the tone presentations show a stable phase relationship as depicted in a representative example recorded from the CA1 and CA3 area at one time point ( $1 \mathrm{~h}$ after the first day of $2 \mathrm{~h}$ stress; Fig. $4 A$ ). The sPLV associated with these trials increases after tone onset (Fig. 4B). We further analyzed this increase by calculating the instantaneous phase differences between AEPs from areas CA1 and CA3 during a $40 \mathrm{~ms}$ period immediately before and $40 \mathrm{~ms}$ after tone onset. There was no phase locking between areas CA1 and CA3 during this pre-tone 
Table 2. Dominant frequencies (mean \pm SEM) of auditory-evoked powers in hippocampal areas CA1 and CA3 and the LA before, during, and after chronic stress

\begin{tabular}{|c|c|c|c|c|c|c|}
\hline & \multicolumn{3}{|c|}{ Dominant frequency $(\mathrm{Hz})$} & \multicolumn{3}{|c|}{$\begin{array}{l}\text { Stress-induced change in power compared with before stress } \\
(\mathrm{Hz}) \text { at } p=0.05\end{array}$} \\
\hline Before stress & $27.7 \pm 1.3$ & $23.4 \pm 1.8$ & $19.8 \pm 1.5$ & & & \\
\hline $24 \mathrm{~h}+(1 \mathrm{~d} \& 2 \mathrm{~d}$ stress $)$ & $27.3 \pm 1.4$ & $24.1 \pm 2.9$ & $26.6 \pm 2.2^{* *}$ & ns & ns & $21-32 \uparrow$ \\
\hline $24 \mathrm{~h}+(9 \mathrm{~d} \& 10 \mathrm{~d}$ stress $)$ & $23.0 \pm 1.5$ & $21.5 \pm 2.0$ & $33.5 \pm 1.8^{* * *}$ & ns & ns & $30-34 \uparrow$ \\
\hline Stress-free recovery & $25.7 \pm 3.6$ & $27.2 \pm 1.9$ & $34.7 \pm 1.3 * * *$ & $23-37 \downarrow$ & $14-39 \downarrow$ & ns \\
\hline
\end{tabular}

${ }^{*} p<0.05,{ }^{* *} p<0.01,{ }^{* * *} p<0.001$ compared with the values before stress. There was a significant main effects of stress (stress-induced change in power) in all three areas on recording time points $\left(\mathrm{CA1}, F_{(4,24)}=9.45, p<0.001 ;(\mathrm{CA} 3\right.$, $\left.F_{(4,24)}=10.19, p<0.001 ; \mathrm{LA}, F_{(4,24)}=7.37, p<0.001\right)$ and frequency $\left(\mathrm{CA1}, F_{(300,1800)}=15.07, p<0.001 ; \mathrm{CA}_{3}, F_{(300,1800)}=11.75, p<0.001 ; \mathrm{LA}, F_{(300,1800)}=3.325, p<0.001\right)($ see Materials and Methods). Arrows indicate directions of change; ns, not significant.

period. However, after the onset of tone (40-80 ms after tone onset), a significant phase locking was observed (Fig. 4C). It is this constant phase difference, caused by phase locking between the two areas, which gives rise to the increase in SPLV (Fig. 4B).

The above analysis was extended to examine the pairwise phase relationship between hippocampal areas CA1 and CA3 and the LA for all time points during and after chronic stress (Fig. 4D-F). Similar to the evoked power, the most pronounced impact of stress on phase synchrony was also seen in different frequency bands within the hippocampus $(30-34 \mathrm{~Hz})$ and between hippocampus and LA (26-36 $\mathrm{Hz})$. There was a significant increase in phase synchrony between areas CA1 and CA3 $1 \mathrm{~h}$ after the first and second sessions of stress $\left[F_{(4,24)}=3.79, p=0.015 ; 1 \mathrm{~h}+(1\right.$ d \& 2 d stress), $p<0.01$; Fig. $4 D$, blue)]. However, this increase was short-lived because it was not evident $24 \mathrm{~h}$ later $[24 \mathrm{~h}+$ (1 d \& 2 d stress); Fig. 4D, orange]. Importantly, when the same stress was repeated for $10 \mathrm{~d}$, there was a decrease in phase synchrony both $24 \mathrm{~h}[24 \mathrm{~h}+(9 \mathrm{~d} \& 10 \mathrm{~d}$ stress), $p<0.05$; Fig $4 D$, red] and $10 \mathrm{~d}$ (stress-free recovery, $p<0.05$; Fig. $4 D$, gray) after the end of chronic stress.

In contrast to the changes seen within the hippocampus, stress had a more persistent impact on phase synchrony between the CA1 area and LA $\left(F_{(4,24)}=4.38, p<0.01\right.$; Fig. $\left.4 E\right)$. There was a significant increase in phase synchrony between these areas $1 \mathrm{~h}$ after the first and second sessions of stress $(p<$ 0.001 ; Fig. $4 E$, blue). Unlike the hippocampus, this increase persisted even $24 \mathrm{~h}$ later $(p<0.05$; Fig. $4 E$, orange). Furthermore, this increase in phase synchrony continued $24 \mathrm{~h}$ after the end of chronic stress $(p<$ 0.05 ; Fig. $4 E$, red) and returned to pre-stress baseline levels only after $10 \mathrm{~d}$ of stress-free recovery (Fig. $4 E$, gray). Unlike areas CA1 and LA, there was a transient increase in phase synchrony between the CA3 area and LA $1 \mathrm{~h}$ after the first and second sessions of stress $\left[F_{(4,24)}=3.408, p=0.024 ; 1 \mathrm{~h}+(1 \mathrm{~d}\right.$ $\& 2$ d stress), $p<0.001$; Fig. $4 F$, blue]. However, this increase was not long lasting.
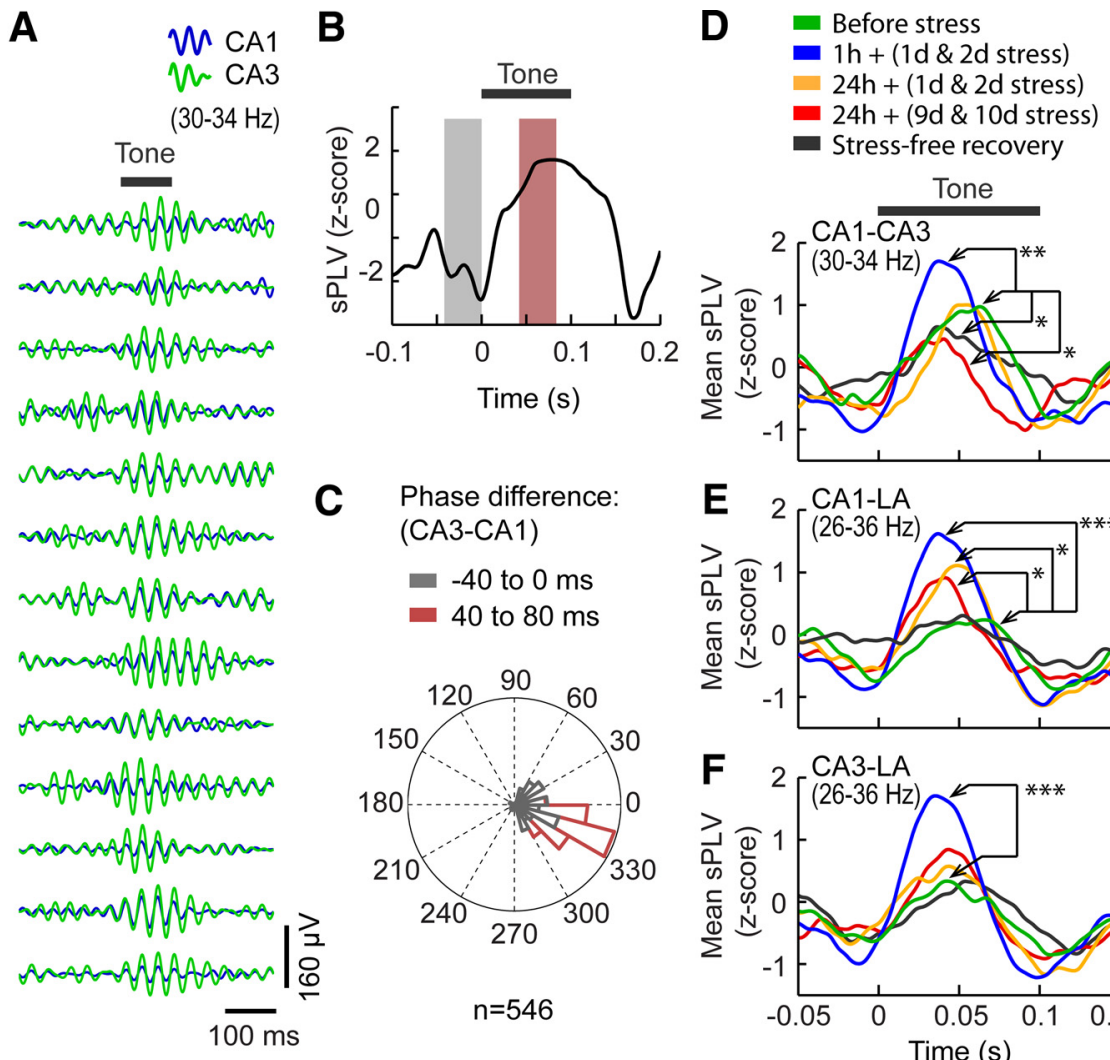

Figure 4. Contrasting effects of chronic stress on phase synchrony in beta/gamma oscillations. $A$, Representative traces of filtered (26-36 Hz) AEPs from areas CA1 (blue) and CA3 (green) $1 \mathrm{~h}$ after first day of stress. $\boldsymbol{B}$, sPLVs computed from these trials in $A$ indicating an increase in phase synchrony after the onset of tone (horizontal bar). $C$, Distribution of the instantaneous phase differences for the above AEP trials before and after the tone-on period ( $40 \mathrm{~ms}$ window marked by gray and pink box; $\boldsymbol{B}$ ), illustrating the emergence of a constant phase relationship after the tone (pink) associated with high sPLV. $\boldsymbol{D}-\boldsymbol{F}$, Average values of sPLV between areas $C A 1$ and $C A 3(30-34 \mathrm{~Hz} ; \boldsymbol{D})$, and $C A 1$ and $L A(26-36 \mathrm{~Hz} ; \boldsymbol{E})$ before, during, and after chronic stress. Phase synchrony in the CA1-CA3 network (D) showed a transient increase [1 h $+(1 \mathrm{~d} \& 2 \mathrm{~d}$ stress), blue; $p<0.01]$ followed by long-lasting impairment after chronic stress [24 $\mathrm{h}+(9 \mathrm{~d} \& 10 \mathrm{~d}$ stress), red, $p<0.05$; stress-free recovery, black, $p<0.05]$. Phase synchrony was persistently enhanced between area CA1 and LA throughout chronic stress [ $1 \mathrm{~h}+(1 \mathrm{~d} \& 2 \mathrm{~d}$ stress), blue, $p<0.001 ; 24 \mathrm{~h}+$ $(1 \mathrm{~d} \& 2 \mathrm{~d}$ stress), orange, $p<0.05 ; 24 \mathrm{~h}+(9 \mathrm{~d} \& 10 \mathrm{~d}$ stress), red, $p<0.05 ; E]$. Between CA3 and LA, there was only a transient increase in the average sPLV $\left[1 \mathrm{~h}+(1 \mathrm{~d} \& 2 \mathrm{~d}\right.$ stress), blue, $p<0.001 ; \boldsymbol{F}] .{ }^{*} p<0.05,{ }^{* *} p<0.01,{ }^{* * *} p<0.001$.

Chronic stress causes a persistent impairment of directional coupling from hippocampal area CA3 to CA1

Stress-induced increase in the power spectra and phase synchrony in beta/gamma oscillations raises the possibility that these oscillations within the amygdala-hippocampal network may be coupled. Indeed, a previous study has reported bidirectional coupling between the hippocampus and amygdala in the theta frequency band after fear conditioning (Popa et al., 2010). Furthermore, a non-zero phase shift in the phase-synchrony analysis together with the negligible amplitude or phase correla- 


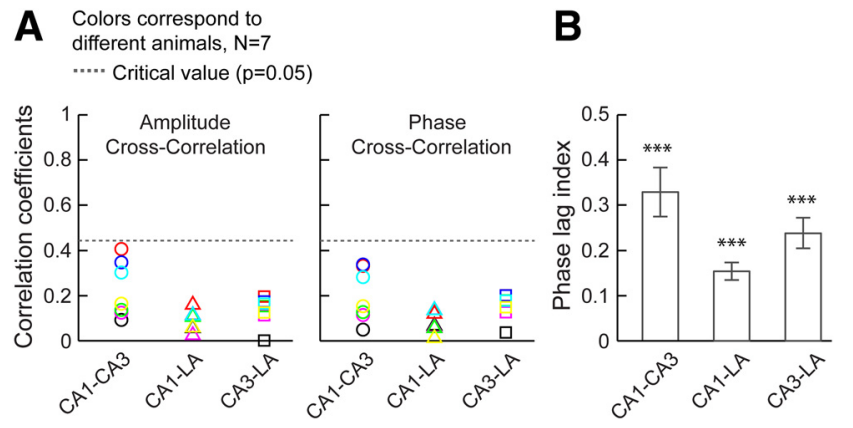

Figure 5. The observed effects were not attributable to contributions from volume conduction between the three recording sites in the hippocampal-amygdalar network. $A$, Crosscorrelations of amplitudes (left) and phases (right) between all pairs of structures, CA1, CA3, and LA before stress within beta/gamma frequency range $(16-36 \mathrm{~Hz})$, indicating nonsignificant contribution of volume conduction in the LFPs. Each color denotes a rat. Dotted lines correspond to the critical values ( 0.44 at $p=0.05$ ). Circles, CA1-CA3; triangles, CA1-LA; squares, CA3-LA. $B$, Mean PLI across rats (see Materials and Methods) for all pairs of brain structures before stress were significantly higher than zero (CA1-CA3, $p<0.001$; CA1-LA, $p<0.001 ; \mathrm{CA} 3-\mathrm{LA}, p<$ $0.001 ; n=7)$, indicating negligible volume conduction. Error bars indicate \pm SEM. ${ }^{* * *} p<$ 0.001 .

tions ( $p<0.05$; Fig. $5 A$ ) as well as significantly high PLI (CA1CA3, $p<0.001$; CA1-LA, $p<0.001$; CA3-LA, $p<0.001$; Fig. $5 B$ ) between CA1, CA3 and LA during spontaneous activity (see Materials and Methods) ruled out the possibility of contributions from volume conduction between the two areas. Therefore, we examined how stress modulates the directionality of these oscillations across the LA-CA1-CA3 network using Granger causality analysis (Granger, 1969; Geweke, 1982). This analysis, which can be performed in the frequency domain, has been used in previous studies in primates and rodents to analyze the directionality of interactions between various brain areas under different behavioral conditions (Brovelli et al., 2004; Gregoriou et al., 2009; Popa et al., 2010). The Granger spectral causality within $16-36 \mathrm{~Hz}$ was computed using a bivariate autoregressive model (50 ms moving window; model order of 15) among all pairs of structures (see Materials and Methods). We observed a dominant directional coupling from area CA $3 \rightarrow$ CA1 before stress $(p<0.01$; Fig. $6 A$, first column). The CA3 $\rightarrow$ CA1 coupling was unaffected 1 and $24 \mathrm{~h}$ after the first and second sessions of stress (Fig. 6A, second and third columns). Interestingly, although the early episodes of stress had no impact on CA3 $\rightarrow$ CA1 coupling, it was significantly impaired $24 \mathrm{~h}$ after the end of the last two sessions of chronic stress [ $24 \mathrm{~h}+(9 \mathrm{~d} \& 10 \mathrm{~d}$ stress), $p<0.05$; Fig. 6 A, fourth column]. This reduction in coupling did not recover even $10 \mathrm{~d}$ after chronic stress ( $p<0.05$; Fig. $6 A$, fifth column).

\section{Directional coupling from the LA to area CA1 is enhanced during and after chronic stress}

Unlike the CA3 $\rightarrow$ CA1 coupling, there was no dominant directional influence between the LA and CA1 area before stress $(p>$ 0.05 ; Fig. $6 B$, first column). However, the initial sessions of stress (first and second sessions) elicited a bidirectional flow between the two areas $1 \mathrm{~h}$ later-a short-latency influence from LA to area CA1 followed by a CA1 $\rightarrow$ LA coupling at a longer latency $[1 \mathrm{~h}+$ ( $1 \mathrm{~d} \& 2 \mathrm{~d}$ stress), $p<0.01$; Fig. $6 B, C$, second column]. However, 24 h later, only the $\mathrm{LA} \rightarrow$ CA1 influence persisted $[24 \mathrm{~h}+(1 \mathrm{~d} \&$ $2 \mathrm{~d}$ stress), $p<0.05$; Fig. $6 B$, third column]. Strikingly, the increase in LA $\rightarrow$ CA1 coupling persisted long enough to be evident not only $24 \mathrm{~h}$ but even $10 \mathrm{~d}$ after chronic stress [ $24 \mathrm{~h}+(9 \mathrm{~d} \& 10 \mathrm{~d}$ stress), $p<0.001$, fourth column; stress-free recovery, $p<0.05$, fifth column; Fig. $6 B$ ]. The latencies of the dominant directional
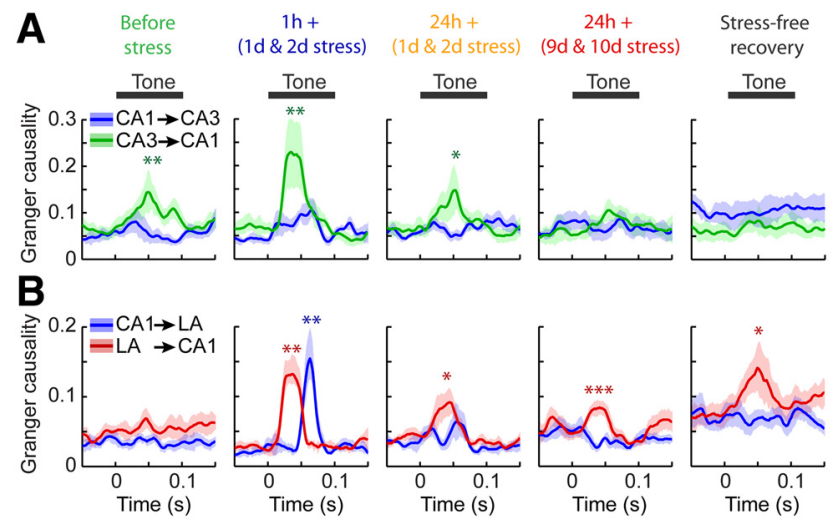

\section{Latency of the dominant directional influence $-\mathrm{CA} 1 \rightarrow \mathrm{CA} 3, \mathrm{LA}=\mathrm{CA} 3 \rightarrow \mathrm{CA} 1=\mathrm{LA} \rightarrow \mathrm{CA} 1$}
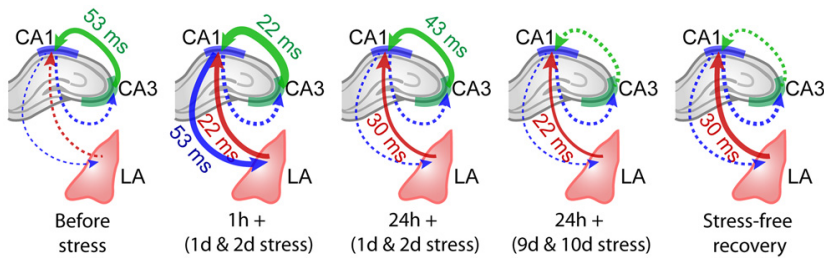

Figure 6. Chronic stress impairs directional coupling from hippocampal areas CA1 to CA3 but enhances it from the LA to CA1. $A, B$, Population averages of Granger causality values (mean \pm SEM) in the beta/gamma band (16-36) calculated for pairwise combinations of AEPs from areas CA1, CA3, and the LA before, during, and after chronic stress. There was a dominant directional influence (arrows) from CA3 to CA1 $(\boldsymbol{A})$ before stress (1st column, $p<0.01$ ) that declined after chronic stress [ $24 \mathrm{~h}+(9 \mathrm{~d} \& 10 \mathrm{~d}$ stress), 4th column; $p>0.05]$ and failed to recover even $10 \mathrm{~d}$ later (stress-free recovery, 5 th column; $p>0.05$ ). Between $L A$ and $C A 1(B)$, there was a transient bidirectional causal entrainment with varying latencies $[1 \mathrm{~h}+(1 \mathrm{~d} \& 2 \mathrm{~d}$ stress), 2nd column; $p<0.01]$. Subsequently, only a unidirectional coupling from LA to CA1 remained strong during and after chronic stress $[24 \mathrm{~h}+(1 \mathrm{~d} \& 2 \mathrm{~d}$ stress $), 3$ rd column; $p<0.05$ $24 \mathrm{~h}+(9 \mathrm{~d} \& 10 \mathrm{~d}$ stress), 4 th column; $p<0.001$; stress-free recovery, 5 th column; $p<0.05]$. C, Granger causality graphs depicting the modulation of directional influence (with latencies) during different stages of stress between the LA and areas CA 1 and CA3. The strength of Granger spectral causality values are coded by the thickness of lines between the three recording sites. The arrowheads indicate the direction of Granger causal influence. Solid and dotted lines indicate presence and absence of dominant directional influence, respectively. ${ }^{*} p<0.05,{ }^{* *} p<$ $0.01,{ }^{* * *} p<0.001$.

coupling during the CIS paradigm are shown in connection diagrams before, during, and after CIS (Fig. 6C).

Similar to the phase synchrony, we did not find any significant effect of chronic stress on the directional coupling from area CA3 to the LA except for a transient increase $1 \mathrm{~h}$ after first and second sessions of stress [before stress, LA $\rightarrow$ CA3, $p<0.01 ; 1 \mathrm{~h}+(1 \mathrm{~d} \&$ 2 d stress), CA3 $\rightarrow$ LA, $p<0.01 ; 24$ h + (1 d \& 2 d stress), LA $\rightarrow$ CA3, $p<0.01 ; 24 \mathrm{~h}+$ (9 d \& $10 \mathrm{~d}$ stress), $p<0.05$; stress-free recovery, LA $\rightarrow$ CA3, $p<0.05$ ].

\section{Discussion}

The present study is one of the first attempts to look at the consequences of stress-induced plasticity in terms of dynamic changes in neuronal oscillations and functional connectivity across the intact hippocampal-amygdalar network in freely behaving rats. A major theme emerging from previous studies is that the same stressful experience elicits contrasting patterns of neural plasticity in the hippocampus and amygdala. However, analyses that led to these findings were mostly restricted in time and space; measurements of stress-induced changes in morphology, biochemical signaling, or synaptic physiology focused on one brain area at a time and at fixed time points after stress (McEwen, 1999; Kim and Diamond, 2002; Roozendaal et al., 


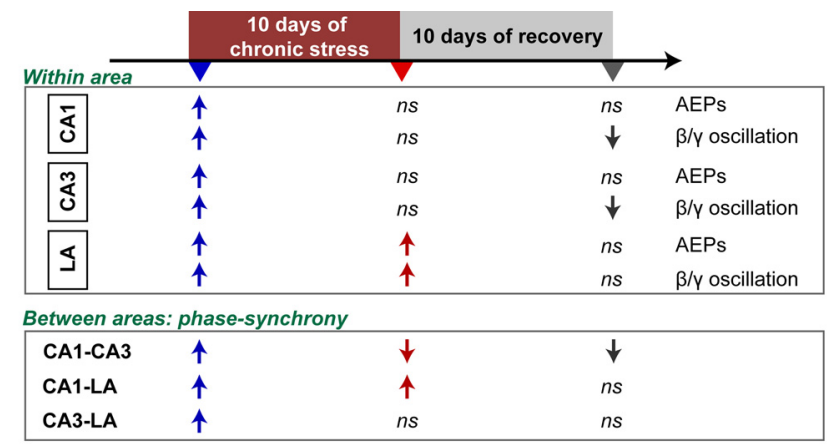

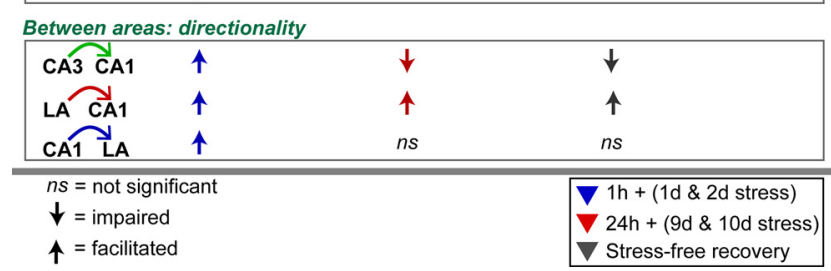

Figure 7. Summary of the divergent effects of chronic stress on auditory evoked neural activity in the hippocampus versus amygdala. The early effects of stress (blue) are similar across the $L A$ and hippocampal areas $C A 1$ and $C A 3$, but differences emerge by the end of chronic stress (red) and during stress-free recovery (gray).

2009). Our findings, based on simultaneous in vivo recordings from three locations in the amygdala and hippocampus, highlight the distinct spatiotemporal dynamics of neural activity across the two areas before, during, and after chronic stress, spanning a total of $21 \mathrm{~d}$ in the same animal (Fig. 7). First, the impact of stress differs in terms of temporal progression and persistence, as well as anatomical location. During the early stages of the $10 \mathrm{~d}$ chronic stress, AEPs are amplified in both the LA and hippocampal areas CA1 and CA3 after one or two episodes of $2 \mathrm{~h}$ acute stress, but differences emerge even during these initial exposures wherein the enhancement seen $1 \mathrm{~h}$ after stress lasts up to $24 \mathrm{~h}$ in the LA and not in the hippocampus. This difference is all the more evident after the same stressor is repeated for $10 \mathrm{~d}$; the enhancement seen in the early sessions habituates in the hippocampus but persists in the amygdala. The same pattern holds for the evoked power of beta/gamma oscillations. Second, stress also has a profound impact on how neuronal assemblies located in the amygdala and hippocampus interact and influence each other over the course of chronic stress. Although beta/gamma synchrony is reduced between areas CA1 and CA3, it increases between the LA and hippocampus after chronic stress. Granger causality analysis of how beta/gamma oscillations flow within the CA3-CA1-LA network also reveals differences: even as repeated exposure to stress weakens CA $3 \rightarrow$ CA 1 coupling within the hippocampus, the directional influence exerted by the LA on the CA1 remains strong throughout the duration of chronic stress and even beyond. Together, these findings add a new dimension to accumulating evidence on the contrasting molecular, synaptic, and behavioral effects of acute and chronic stress on the amygdala and hippocampus.

\section{Similarities in the immediate effects of acute stress}

Despite the gradual emergence of differences over the course of the chronic stress, the initial response to stress is remarkably similar in the amygdala and hippocampus. The AEP amplitudes and beta/gamma evoked power are significantly enhanced in all three recording sites $1 \mathrm{~h}$ after the first and second exposures to acute stress. This is consistent with reports that stress enhances extracellular levels of glutamate in both the hippocampus and amygdala (Reznikov et al., 2007). Elevation in corticosterone levels also elicits transient increase in neuronal excitability in CA1 pyramidal neurons (Weiss et al., 2005). Similar to the hippocampus, acute stress increases glutamate efflux and reduces GABAergic inhibition in the BLA (Reznikov et al., 2007, 2009). Interestingly, in vitro application of stress levels of corticosterone decreases GABAergic transmission and increases excitability of LA principal neurons (Duvarci and Paré, 2007). Consistent with this, acute stress and corticosterone treatment enhance baseline responses and LTP in the BLA in vivo (Vouimba et al., 2004; Kavushansky and Richter-Levin, 2006). Together, these changes are likely to increase in excitatory drive that enhances AEPs and beta/gamma power seen in both the hippocampus and amygdala. Interestingly, the initial increase in AEP amplitude did not persist in the amygdala $10 \mathrm{~d}$ after the cessation of chronic stress. This is surprising because amygdalar dendritic hypertrophy persists up to $21 \mathrm{~d}$ after chronic stress (Vyas et al., 2004). This suggests that short-term physiological modulation of neuronal excitability caused by changes in corticosterone, glutamate, or GABA rather than stress-induced dendritic growth in BLA neurons may have a greater impact on AEPs.

\section{Emergence of divergent effects and weakening of the CA3-CA1 network}

Although the immediate consequences of stress are similar in both brain areas, there is growing evidence that subsequently these areas exhibit contrasting patterns of plasticity with prolonged stress (Roozendaal et al., 2009). Thus, cellular mechanisms more downstream of these short-term changes may underlie the differential response of the two brain structures to the same chronic stress. In this connection, much of the previous work has assessed morphological and electrophysiological changes at the end of chronic stress. Consistent with these effects, we find that synchrony between areas CA3 and CA1, and the directional influence exerted by the former on the latter, are weakened by chronic stress. Interestingly, enhanced CA3-CA1 gamma phase synchronization has been observed just before rodents make a decision about the choice of direction in a T-maze (Montgomery and Buzsáki, 2007). Chronic restraint stress, in turn, has been shown to impair spatial memory in the T- and Y-mazes (Conrad, 2010). Thus, impairment of phase synchrony within the CA3-CA1 network is likely to contribute to poor performance in spatial and working memory tasks after chronic stress.

\section{Stronger directional coupling from the amygdala to the hippocampus}

We also find that the same chronic stress that disrupts synchrony in the CA3-CA1 network ends up enhancing it between the LA and CA1 area. In addition to the contrasting morphological effects of stress on the hippocampus versus amygdala, restraint stress causes GABAergic disinhibition and reduces the threshold for LTP induction in BLA slices (Rodríguez Manzanares et al., 2005). Consistent with these divergent effects spanning multiple levels of neural organization reported previously, we find that both AEPs and the power of beta/gamma oscillations are persistently enhanced in the LA but not in the hippocampus throughout the $10 \mathrm{~d}$ of chronic stress. This is likely to tilt the balance toward stronger encoding of emotionally salient memories. Indeed, this is in agreement with behavioral studies showing that repeated stress facilitates amygdala-dependent fear learning (Shors et al., 1992). 


\section{Functional implications and future directions}

What may be the behavioral consequences of the gradual and persistent increase in the directional influence exerted by the amygdala over the hippocampus? A study (Conrad et al., 1999) highlighting the importance of the amygdala reported that repeated restraint stress facilitates fear conditioning to both context (depends on the hippocampus and amygdala) and tone (depends only on the amygdala). Surprisingly, although the antidepressant tianeptine prevented stress-induced neuronal atrophy in the hippocampus, it failed to prevent the increase in contextual fear conditioning, raising the possibility that chronic stress may have a powerful impact on the amygdala that could prevail over any influence of the hippocampus. Consistent with this, Granger causality analysis uncovers a robust directional influence from the LA to area CA1 that remains strong even after stress-free recovery. Indeed, there is growing evidence supporting such a mechanism. First, neuroanatomical data point to extensive direct and indirect interconnections between the LA/BLA and area CA1 (McDonald, 1998; Pikkarainen et al., 1999). Second, both lesions and pharmacological inactivation of the BLA prevent stressinduced impairment of hippocampal LTP and spatial memory in rats (Kim et al., 2001, 2005). Third, in vivo electrical activation of the amygdala, but not corticosterone treatment, mimics the effects of stress on CA1 place cells (Kim et al., 2012). These observations, together with our results, suggest that the amygdala is in a position to play a significant role in mediating the effects of stress on hippocampal function.

In conclusion, our findings suggest that amygdalar influence on the hippocampus becomes dominant during chronic stress. In particular, stress appears to shift control over area CA1 from the CA3 area to LA, which may explain some of the previously reported effects of stress on hippocampal place cells and spatial learning and memory (Kim et al., 2007). Importantly, some of these changes persist even $10 \mathrm{~d}$ after the cessation of stress, raising the possibility of a prolonged rewiring of the amygdala-hippocampal network. However, it is important to note that Granger causality analysis is a statistical measure, and functional connectivity suggested by such analysis in itself does not mean that the amygdala is exerting its influence directly through axonal inputs to the hippocampus. Thus, future studies will be needed to investigate the role of amygdalar projection neurons and their outputs in modulating stress-induced plasticity mechanisms in the hippocampus. The effects of stress on amygdala neurons have been characterized in considerable detail, but little is known about their projection identities. For instance, do the amygdala neurons that undergo enhancement in physiological and morphological plasticity after stress project directly to those hippocampal pyramidal neurons in areas CA1 and CA3 that undergo stressinduced plasticity in the opposite direction? If so, do long-range anatomical connections between these distinct neuronal populations in the two areas mediate the functional interactions suggested by the patterns of Granger causality reported here? Answers to these questions are likely to provide new insights into how aberrant interactions across the amygdala-hippocampal network, not just those confined within each area, leads to disruption of cognitive and emotional function in stress-related psychiatric disorders.

\section{References}

Brockhaus-Dumke A, Mueller R, Faigle U, Klosterkoetter J (2008) Sensory gating revisited: relation between brain oscillations and auditory evoked potentials in schizophrenia. Schizophr Res 99:238-249. CrossRef Medline
Brovelli A, Ding M, Ledberg A, Chen Y, Nakamura R, Bressler SL (2004) Beta oscillations in a large-scale sensorimotor cortical network: directional influences revealed by Granger causality. Proc Natl Acad Sci USA 101:9849-9854. CrossRef Medline

Conrad CD (2010) A critical review of chronic stress effects on spatial learning and memory. Prog Neuropsychopharmacol Biol Psychiatry 34:742755. CrossRef Medline

Conrad CD, LeDoux JE, Magariños AM, McEwen BS (1999) Repeated restraint stress facilitates fear conditioning independently of causing hippocampal CA3 dendritic atrophy. Behav Neurosci 113:902-913. CrossRef Medline

Cui J, Xu L, Bressler SL, Ding M, Liang H (2008) 2008 Special issue: BSMART: a Matlab/C toolbox for analysis of multichannel neural time series. Neural Networks 21:1094-1104. CrossRef Medline

Daffertshofer A, Stam C (2007) Influences of volume conduction on phase distributions. Int Congress Series 1300:209-212. CrossRef

Ding M, Bressler SL, Yang W, Liang H (2000) Short-window spectral analysis of cortical event-related potentials by adaptive multivariate autoregressive modeling: data preprocessing, model validation, and variability assessment. Biol Cybern 83:35-45. CrossRef Medline

Duvarci S, Paré D (2007) Glucocorticoids enhance the excitability of principal basolateral amygdala neurons. J Neurosci 27:4482-4491. CrossRef Medline

Dzirasa K, Ramsey AJ, Takahashi DY, Stapleton J, Potes JM, Williams JK, Gainetdinov RR, Sameshima K, Caron MG, Nicolelis MA (2009) Hyperdopaminergia and NMDA receptor hypofunction disrupt neural phase signaling. J Neurosci 29:8215-8224. CrossRef Medline

Geweke J (1982) Measurement of linear dependence and feedback between multiple time series. J Am Stat Assoc 77:304-313. CrossRef

Granger CW (1969) Investigating causal relations by econometric models and cross-spectral methods. Econometrica 37:424-438.

Gregoriou GG, Gotts SJ, Zhou H, Desimone R (2009) High-frequency, long-range coupling between prefrontal and visual cortex during attention. Science 324:1207-1210. CrossRef Medline

Haenschel C, Baldeweg T, Croft RJ, Whittington M, Gruzelier J (2000) Gamma and beta frequency oscillations in response to novel auditory stimuli: a comparison of human electroencephalogram (EEG) data with in vitro models. Proc Natl Acad Sci USA 97:7645-7650. CrossRef Medline

Jung J, Hudry J, Ryvlin P, Royet JP, Bertrand O, Lachaux JP (2006) Functional significance of olfactory-induced oscillations in the human amygdala. Cereb Cortex 16:1-8. CrossRef Medline

Kavushansky A, Richter-Levin G (2006) Effects of stress and corticosterone on activity and plasticity in the amygdala. J Neurosci Res 84:1580-1587. CrossRef Medline

Kim EJ, Kim ES, Park M, Cho J, Kim JJ (2012) Amygdalar stimulation produces alterations on firing properties of hippocampal place cells. J Neurosci 32:11424-11434. CrossRef Medline

Kim JJ, Diamond DM (2002) The stressed hippocampus, synaptic plasticity and lost memories. Nat Rev Neurosci 3:453-462. CrossRef Medline

Kim JJ, Lee HJ, Han JS, Packard MG (2001) Amygdala is critical for stressinduced modulation of hippocampal long-term potentiation and learning. J Neurosci 21:5222-5228. Medline

Kim JJ, Koo JW, Lee HJ, Han JS (2005) Amygdalar inactivation blocks stress-induced impairments in hippocampal long-term potentiation and spatial memory. J Neurosci 25:1532-1539. CrossRef Medline

Kim JJ, Lee HJ, Welday AC, Song E, Cho J, Sharp PE, Jung MW, Blair HT (2007) Stress-induced alterations in hippocampal plasticity, place cells, and spatial memory. Proc Natl Acad Sci USA 104:18297-18302. CrossRef Medline

Lachaux JP, Rodriguez E, Michel LV, Antoine L, Martinerie J, Francisco JV (2000) Studying single-trials of phase synchronous activity in the brain. Int J Bifurcation Chaos 10:2429-2439. CrossRef

Li X, Yao X, Fox J, Jefferys JG (2007) Interaction dynamics of neuronal oscillations analysed using wavelet transforms. J Neurosci Methods 160: 178-185. CrossRef Medline

Luine V, Villegas M, Martinez C, McEwen BS (1994) Repeated stress causes reversible impairments of spatial memory performance. Brain Res 639: 167-170. CrossRef Medline

Makeig S, Debener S, Onton J, Delorme A (2004) Mining event-related brain dynamics. Trends Cogn Sci 8:204-210. CrossRef Medline

Rodríguez Manzanares PAR, Isoardi NA, Carrer HF, Molina VA (2005) Previous stress facilitates fear memory, attenuates GABAergic inhibition, 
and increases synaptic plasticity in the rat basolateral amygdala. J Neurosci 25:8725-8734. CrossRef Medline

McDonald AJ (1998) Cortical pathways to the mammalian amygdala. Prog Neurobiol 55:257-332. CrossRef Medline

McEwen BS (1999) Stress and hippocampal plasticity. Annu Rev Neurosci 22:105-122. CrossRef Medline

Montgomery SM, Buzsáki G (2007) Gamma oscillations dynamically couple hippocampal CA3 and CA1 regions during memory task performance. Proc Natl Acad Sci USA 104:14495-14500. CrossRef Medline

Pavlides C, Nivón LG, McEwen BS (2002) Effects of chronic stress on hippocampal long-term potentiation. Hippocampus 12:245-257. CrossRef Medline

Pawlak R, Rao BS, Melchor JP, Chattarji S, McEwen B, Strickland S (2005) Tissue plasminogen activator and plasminogen mediate stress-induced decline of neuronal and cognitive functions in the mouse hippocampus. Proc Natl Acad Sci USA 102:18201-18206. CrossRef Medline

Paxinos G, Watson C (2005) The rat brain in stereotaxic coordinates, 5th ed. London: Elsevier Academic.

Pikkarainen M, Rönkkö S, Savander V, Insausti R, Pitkänen A (1999) Projections from the lateral, basal, and accessory basal nuclei of the amygdala to the hippocampal formation in rat. J Comp Neurol 403:229-260. CrossRef Medline

Pitkänen A, Pikkarainen M, Nurminen N, Ylinen A (2000) Reciprocal connections between the amygdala and the hippocampal formation, perirhinal cortex, and postrhinal cortex in rat: a review. Ann NY Acad Sci 911: 369-391. CrossRef Medline

Popa D, Duvarci S, Popescu AT, Léna C, Paré D (2010) Coherent amygdalocortical theta promotes fear memory consolidation during paradoxical sleep. Proc Natl Acad Sci USA 107:6516-6519. CrossRef Medline

Reznikov LR, Grillo CA, Piroli GG, Pasumarthi RK, Reagan LP, Fadel J (2007) Acute stress-mediated increases in extracellular glutamate levels in the rat amygdala: differential effects of antidepressant treatment. Eur J Neurosci 25:3109-3114. CrossRef Medline

Reznikov LR, Reagan LP, Fadel JR (2009) Effects of acute and repeated restraint stress on GABA efflux in the rat basolateral and central amygdala. Brain Res 1256:61-68. CrossRef Medline
Rogan MT, Stäubli UV, LeDoux JE (1997) Fear conditioning induces associative long-term potentiation in the amygdala. Nature 390:604-607. CrossRef Medline

Roozendaal B, McEwen BS, Chattarji S (2009) Stress, memory and the amygdala. Nat Rev Neurosci 10:423-433. CrossRef Medline

Seidenbecher T, Laxmi TR, Stork O, Pape HC (2003) Amygdalar and hippocampal theta rhythm synchronization during fear memory retrieval. Science 301:846-850. CrossRef Medline

Shors TJ, Weiss C, Thompson RF (1992) Stress-induced facilitation of classical conditioning. Science 257:537-539. CrossRef Medline

Spencer KM, Niznikiewicz MA, Shenton ME, McCarley RW (2008) Sensory-evoked gamma oscillations in chronic schizophrenia. Biol Psychiat 63:744-747. CrossRef Medline

Tang J, Wagner S, Schachner M, Dityatev A, Wotjak CT (2003) Potentiation of amygdaloid and hippocampal auditory-evoked potentials in a discriminatory fear-conditioning task in mice as a function of tone pattern and context. Eur J Neurosci 18:639-650. CrossRef Medline

Uhlhaas PJ, Singer W (2010) Abnormal neural oscillations and synchrony in schizophrenia. Nat Rev Neurosci 11:100-113. CrossRef Medline

Vouimba RM, Yaniv D, Diamond D, Richter-Levin G (2004) Effects of inescapable stress on LTP in the amygdala versus the dentate gyrus of freely behaving rats. Eur J Neurosci 19:1887-1894. CrossRef Medline

Vyas A, Mitra R, ShankaranarayanaRao BS, Chattarji S (2002) Chronic stress induces contrasting patterns of dendritic remodeling in hippocampal and amygdaloid neurons. J Neurosci 22:6810-6818. Medline

Vyas A, Pillai AG, Chattarji S (2004) Recovery after chronic stress fails to reverse amygdaloid neuronal hypertrophy and enhanced anxiety-like behavior. Neuroscience 128:667-673. CrossRef Medline

Weiss C, Sametsky E, Sasse A, Spiess J, Disterhoft JF (2005) Acute stress facilitates trace eyeblink conditioning in C57BL/6 male mice and increases the excitability of their CA1 pyramidal neurons. Learn Mem 12: 138-143. CrossRef Medline

Wiener N, Beckenbach E (1956) Modern mathematics for engineers. New York: McGraw-Hill. 